\title{
On a characterization of finite groups of p-rank 1
}

\author{
By \\ Goro NishidA \\ Dedicated to Professor A. Komatu on his 70th birthday \\ (Received Sept. 13, 1977)
}

\section{\$1. Introduction and statement of the results}

Let $G$ be a finite group. Let $p$ be a prime number. Define the $p$-rank $r_{p}(G)$ of $G$ by the maximal integer $k$ such that $G$ contains the elementary abelian $p$-group $\left(Z_{p}\right)^{k}$ of rank $k$.

It is obvious that $G$ is of $p$-rank 0 if and only if the $p$-Sylow subgroup $G_{(p)}=e$. According to Cartan-Eilenberg [5], we see that $G$ is of $p$-rank 1 if and only if $G_{(p)}$ is either a cyclic group $Z_{p^{r}}$ or a generalized quoternionic group if $p=2$. It is also shown [5] that a finite group $G$ with $p$-rank 0 or 1 for any $p$ is characterized by having the periodic cohomology. Such a group is called an Artin-Tate group.

Now the purpose of the present note is to give a characterization of finite groups of $p$-rank 1 in terms of stable homotopy groups.

Let $|G|$ be the order of $G$ and let $\Sigma_{n}$ denote the symmetric group on $n$ letters. We denote by $\rho=\rho_{G}: G \rightarrow \Sigma_{|G|}$ the regular permutation representation, and $B \rho$ : $B G \rightarrow B \Sigma_{|G|}$ denotes the induced map on classifying spaces. Let

$$
\text { (1): } \coprod_{n} B \Sigma_{n} \longrightarrow \Omega B\left(\bigsqcup_{n} B \Sigma_{n}\right) \simeq Q\left(S^{0}\right)
$$

be the Barratt-Priddy-Quillen map [3], where $Q\left(S^{0}\right)=\lim _{k} \Omega^{k} S^{k}$. Then as the adjoint of the composition

$$
B G_{+} \stackrel{B \rho_{+}}{\longrightarrow} B \Sigma_{|G|+} \subset \coprod_{n} B \Sigma_{n} \stackrel{\omega}{\longrightarrow} Q\left(S^{0}\right)
$$

we obtain a stable map of spectra

$$
f: \mathbf{S}\left(B G_{+}\right) \longrightarrow \mathbf{S}
$$

where $B G_{+}=B G \cup$ disjoint base point. Then we obtain a homomorphism

$$
\phi=\phi_{G}: \pi_{n}^{\mathbf{S}}\left(B G_{+}\right) \longrightarrow \pi_{n}^{\mathbf{S}}\left(S^{0}\right)
$$

of stable homotopy groups. Note that $\pi_{n}^{\mathbf{S}}\left(B G_{+}\right) \cong \pi_{n}^{\mathbf{S}}(B G) \oplus \pi_{n}^{\mathbf{S}}\left(S^{0}\right)$, direct sum. The restriction $\left.\phi\right|_{\pi_{n}(B G)}$ is also denoted by $\phi$.

Now let $J: \pi_{n}(O) \rightarrow \pi_{n}^{\mathbf{S}}\left(S^{0}\right)$ denote the $J$-homomorphism, where $O=\lim O(n)$. 
Restricting $J: \pi_{n}(O) \rightarrow \pi_{n}^{\mathrm{S}}\left(S^{0}\right)$ on $\pi_{n}(U)$ or $\pi_{n}\left(S_{p}\right)$, we obtain the complex $J$-homomorphism $J_{C}$ or the quoternionic $J$-homomorphism $J_{I !}$.

For a finite abelian group $A$, we denote by $A_{(p)}$ the $p$-component of $A$. Then we can state our theorems.

Theorem 1.1. Let $G$ be a finite group of p-rank 1 . If $p$ is odd, then

$$
\operatorname{Im}\left[\phi: \pi_{*}^{\mathrm{s}}(B G) \longrightarrow \pi_{*}^{\mathrm{S}}\left(S^{0}\right)\right] \supset(\operatorname{Im} J)_{(p)}=\left(\operatorname{Im} J_{C}\right)_{(p)} .
$$

If $p=2$, then

$$
\operatorname{Im}\left[\phi: \pi_{*}^{\mathrm{s}}(B G) \longrightarrow \pi_{*}^{\mathrm{s}}\left(S^{0}\right)\right] \supset\left(\operatorname{Im} J_{H}\right)_{(2)} .
$$

Theorem 1.2. Let $G$ be a finite group. Then the p-rank of $G$ is equal to 1 if and only if $\phi: \pi_{2 p-3}^{\mathbf{S}}(B G)_{(p)} \rightarrow \pi_{2 p-3}^{\mathbf{S}}\left(S^{0}\right)_{(p)}\left(\phi: \pi_{3}^{\mathbf{S}}(B G)_{(2)} \rightarrow \pi_{3}^{\mathbf{S}}\left(S^{0}\right)_{(2)}\right.$ if $\left.p=2\right)$ is an epimorphism.

Concerning with the 2-component, it may be worth showing the following

Proposition 1.3. $\phi: \pi_{1}^{\mathbf{S}}(B G) \rightarrow \pi_{1}^{\mathrm{s}}\left(S^{0}\right)$ is an epimorphism if and only if the 2-Sylow subgroup $G_{(2)}$ is a non trivial cyclic group.

From this proposition it follows immediately that if $G_{(2)}$ is non trivial cyclic, then $G$ is not perfect. hence not simple unless $G=Z_{2}$ (Burnside's theorem).

If one uses the Feit-Thompson theorem [6], one can show the following

Corollary 1.4. Let $G$ be an Artin-Tate group. Suppose that $H_{i}(G: \mathbf{Z})=$ $0,1 \leq i \leq 3$, then $G$ is trivial.

Proof. By the assumption, $\pi_{3}^{\mathrm{S}}(B G)=0$. Hence by Theorem 1.2, we see that $G_{(2)}=e$, i.e., $G$ is of odd order. Then by the Feit-Thompson theorem, $G$ is solvable. Then $H_{1}(G: \mathbf{Z})=0$ implies $G=e$.

q.e.d.

Now for a finite group $G$ of $p$-rank 1, Theorem 1.1 shows the non-triviality of $\pi_{2 p-3}^{\mathrm{S}}(B G)_{(p)}\left(\pi_{3}^{\mathrm{S}}(B G)_{(2)}\right.$ if $\left.p=2\right)$. We remark that such a non-triviality of $\pi_{i}^{\mathrm{S}}(B G)_{(p)}$ for $i<2 p-3$ does not hold as the following examples show. If $p$ is odd, then $\Sigma_{p}$ is of p-rank 1. It is known [10] that $H_{i}\left(B \Sigma_{p}: Z_{p}\right)=0$ for $i<2 p-3$. Then by Serre's class theory, $\pi_{i}^{\mathbf{s}}\left(B \Sigma_{p}\right)_{(p)}=0$ if $i<2 p-3$. For $p=2$, consider the binary icosahedral group $I^{*}$. This is a subgroup of order 120 of $S p(1)=S^{3}$. Hence $I^{*}$ is an ArtinTate group and $I_{(2)}^{*}$ is the quoternionic group. It is well-known [16] that $H_{1}\left(B I^{*}\right)$ $=H_{2}\left(B I^{*}\right)=0$. Hence $\pi_{i}^{\mathrm{s}}\left(B I^{*}\right)=0$ for $i \leq 2$.

The non-triviality of $\pi_{2 p-3}^{\mathrm{s}}(B G)_{(p)}\left(\pi_{3}^{\mathrm{s}}(B G)_{(2)}\right)$ clearly fails for general finite groups as the following Quillen's example shows. Let $\mathbf{F}_{q}$ be the finite field with $q=p^{d}$ elements. Then Quillen has shown [11] that $H^{i}\left(B G L\left(n, \mathbf{F}_{q}\right): Z_{p}\right)=0$ for $0<i<d(p-1)$. Thus $\pi_{i}^{\mathbf{S}}\left(B G L\left(n, \mathbf{F}_{q}\right)\right)_{(p)}=0$ for $i<d(p-1)$.

For a cyclic group $Z_{p}$ of prime order, Theorem 1.1 is a direct consequence of the Kahn-Priddy theorem [7], that is $\phi: \pi_{*}^{\mathbf{S}}\left(B Z_{p}\right) \rightarrow \pi_{*}^{\mathbf{S}}\left(S^{0}\right)_{(p)}$ is an epimorphism $(*>0)$. We shall show that the Kahn-Priddy theorem fails for cyclic group of order $2^{r}, r \geq 2$. 
Theorem 1.5. Let $r$ be an integer $\geq 2$. Let $f: \mathbf{S} B Z_{2^{r}} \rightarrow \mathbf{S}$ be an arbitrary stable map. Then $f_{*}: \pi_{7}^{\mathbf{S}}\left(B Z_{2^{r}}\right) \rightarrow \pi_{7}^{\mathbf{S}}\left(S^{0}\right)_{(2)}$ is not epimorphism.

For an odd prime, the problem seems to be more difficult. For example, a direct computation shows that the element $\beta_{1} \in \pi_{2 p(p-1)-2}^{\mathrm{s}}\left(S^{0}\right)_{(p)}$ is in the image of $\phi: \pi_{*}^{\mathbf{S}}\left(B Z_{p^{r}}\right) \rightarrow \pi_{*}^{\mathbf{S}}\left(S^{0}\right)$ for any $r$.

The proof of Theorem 1.1, 1.2 and Proposition 1.3 will be given in $\S 3$, and that of Theorem 1.5 in $\S 4$.

\section{\$2. Factorization of the J-homomorphism}

In this section, we review some results of Becker and Schultz [4]. For odd primary component, similar results can be obtained based on the algebraic $K$-theory which will be explained in the Appendix.

Let $G$ be a finite group. Suppose that $G$ has a free orthogonal representation $W(G$ acts freely on the unit sphere $S(W))$. Let $k W=W \oplus \cdots \oplus W$, the direct sum of $k$ copies of $W$. Set

$$
F_{G}=\lim _{k} M(S(k W), S(k W)),
$$

where $M($,$) denotes the set of G$-maps with compact open topology.

For a topological space $X$, let $Q(X)=\lim _{k} \Omega^{k} S^{k} X$. Then one has

Theorem 2.1. ([4]). There is a homotopy equivalence

$$
\lambda=\lambda_{G}: F_{G} \longrightarrow Q\left(B G_{+}\right) .
$$

For the naturality of $\lambda_{G}$ with respect to $G$, one can see the following. Let $H$ be a subgroup of $G$. Then there is a map

$$
\psi: F_{G} \longrightarrow F_{H}
$$

by regarding $G$-maps as $H$-maps. Next consider the finite covering map $B H \rightarrow B G$. According to Kahn and Priddy [7], one can associate to the finite covering a map

$$
\tau: Q\left(B G_{+}\right) \longrightarrow Q\left(B H_{+}\right)
$$

called the transfer map for $B H \rightarrow B G$. Then we have $([4], 6.10)$.

Proposition 2.2. The following diagram is homotopy commutative

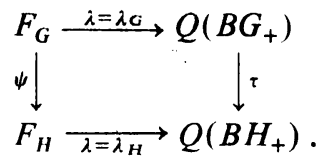

For the homotopy functor $\left[, F_{G}\right]$ and $\left[, Q\left(B G_{+}\right)\right]$, induced maps of maps in the above diagram are denoted by the same letter, e.g.,

$$
\lambda:\left[, F_{G}\right] \longrightarrow\left[, Q\left(B G_{+}\right)\right] .
$$


Now let us recall the definition of the transfer of Kahn and Priddy [7]. Let $k=[G, H]$. The left coset $G / H$ is a $G$-set of order $k$ with the standard left $G$-action. Let $g_{1}, \ldots, g_{k}$ be a representatives of the coset $G / H$. Then the $G$-action on $G / H$ determines a homomorphism

$$
\gamma: G \longrightarrow \Sigma_{k}
$$

by the formula $g g_{i}=g_{\gamma(g)(i)} h_{i}, h_{i} \in H$. Define a homomorphism

$$
\mu: G \longrightarrow \Sigma_{k} \int H \text {. }
$$

by $\mu(g)=\left(\gamma(g) ; h_{1}, \ldots, h_{k}\right)$, where $\Sigma_{k} \int H$ denotes the wreath product. Let

$$
B \mu: B G \longrightarrow B\left(\Sigma_{k} \int H\right)
$$

be the induced map on classifying spaces. Note that $B\left(\Sigma_{k} \int H\right)$ is identified with $E \Sigma_{k} \otimes_{\Sigma_{k}}(B H)^{k}$, where $E \Sigma_{k}$ is a universal $\Sigma_{k}$-space and $\Sigma_{k}$ acts on $(B H)^{k}$ by permutations of factors. It is known (see, e.g., [9]) that there is a canonical map

$$
\omega: \bigsqcup_{n} E \Sigma_{n} \times_{\Sigma_{n}}(B H)^{n} \longrightarrow Q\left(B H_{+}\right) .
$$

Then the composition

$$
B G_{+} \stackrel{B \mu}{\longrightarrow}\left(E \Sigma_{k} \times_{\Sigma_{k}}(B H)^{k}\right)_{+} \subset \coprod_{n} E \Sigma_{n} \times_{\Sigma_{n}}(B H)^{n} \stackrel{\omega}{\longrightarrow} Q\left(B H_{+}\right)
$$

extends to the transfer map.

$$
\tau: Q\left(B G_{+}\right) \longrightarrow Q\left(B H_{+}\right)
$$

using the natural transformation $Q Q \rightarrow Q$ (see [7]).

The following lemma about the functoriality follows from the definition and a straightforward argument.

Lemma 2.3. Let $H \supset K$ be subgroups of $G$. Then the following diagram is homotopy commutative

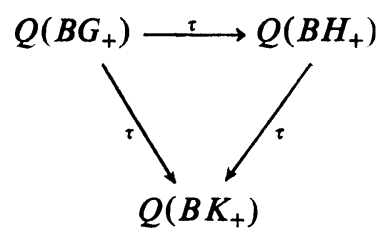

Suppose now that $H=e$. Then $\tau$ is clearly the extension of

$$
B G_{+} \stackrel{B \rho}{\longrightarrow} B \Sigma_{|G|} \stackrel{\omega}{\longrightarrow} Q\left(S^{0}\right) .
$$

hence $\tau_{*}: \pi_{i}\left(Q\left(B G_{+}\right)\right) \cong \pi_{i}^{\mathbf{S}}\left(B G_{+}\right) \rightarrow \pi_{i}\left(Q\left(S^{0}\right)\right) \cong \pi_{i}^{\mathbf{S}}\left(S^{0}\right)$ is just the homomorphism $\phi$ defined in $\$ 1$. 
Consider the homomorphism $\phi: \pi_{*}^{\mathbf{S}}\left(B G_{+}\right) \cong \pi_{*}^{\mathbf{S}}(B G) \oplus \pi_{*}^{\mathbf{S}}\left(S^{0}\right) \rightarrow \pi_{*}^{\mathbf{S}}\left(S^{0}\right)$. Then we have

Lemma 2.4. $\left.\phi\right|_{\pi_{*}^{\mathbf{S}}\left(S^{0}\right)}: \pi_{*}^{\mathbf{S}}\left(S^{0}\right) \rightarrow \pi_{*}^{\mathbf{S}}\left(S^{0}\right)$ is the multiplication with $|G|$.

Proof. $\left.\phi\right|_{\pi_{*}^{\mathbf{s}}\left(S^{0}\right)}$ is induced from the adjoint of the composition map

$$
g: S^{0} \stackrel{i}{\longrightarrow} B G_{+} \longrightarrow B \Sigma_{|G|+} \longrightarrow Q\left(S^{0}\right)
$$

where $i: S^{0} \rightarrow B G_{+}$maps the non base point of $S^{0}$ into $B G$. Hence $g$ maps that point into the component of degree $|G|$ maps of $Q\left(S^{0}\right)$. Hence $\operatorname{Ad}(g): \mathbf{S} \rightarrow \mathbf{S}$ is a map of degree $|G|$. This shows the lemma.

q.e.d.

Let $K=\mathbf{R}, \mathbf{C}$ or $\mathbf{H}$ be the field of real, complex or quoternionic numbers, respectively. Let $O_{K}(n)$ denote $O(n), U(n)$ or $S p(n)$ according to $K=\mathbf{R}, \mathbf{C}$ or $\mathbf{H}$, respectively. We let $O_{K}(1)\left(=Z_{2}, S^{1}\right.$ or $\left.S^{3}\right)$ act on $K^{n}$ as the scalar multiplication. Then any element $f \in O_{K}(n)$ gives an $O_{K}(1)$-equivariant map $f: S\left(K^{n}\right) \rightarrow S\left(K^{n}\right)$. Let $G$ be a finite subgroup of $O_{K}(1)$. Then we obtain a map

$$
j_{G}: O_{K}(\infty) \longrightarrow F_{G} .
$$

By Theorem 2.1, there is an isomorphism $\lambda:\left[, F_{G}\right] \cong\left[, Q\left(B G_{+}\right)\right]$. Hence $j_{G}$ induces a map

$$
j_{G}:\left[, O_{K}(\infty)\right] \longrightarrow\left[, Q\left(B G_{+}\right)\right] .
$$

It is obvious that if $G=e$, then

$$
j_{e}:\left[, O_{K}(\infty)\right] \longrightarrow\left[, Q\left(S^{0}\right)\right]
$$

agrees with the $(K-) J$-homomorphism.

If $G \supset H$, then we see easily that

$$
\psi j_{G}=j_{H}: O_{K}(\infty) \longrightarrow F_{H}
$$

Then setting $H=e$, we have obtained

Proposition 2.5. The following diagram is commutative.

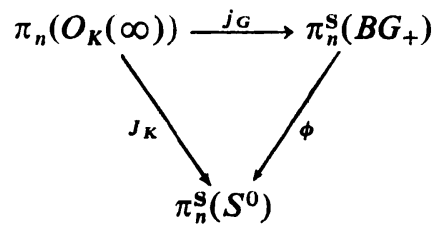

\section{§3. Finite groups of p-rank 1}

Proposition 3.1. Let $p$ be a prime number and $a \geq 1$ an integer. Then

$$
\operatorname{Im}\left[\phi: \pi_{*}^{\mathbf{S}}\left(B Z_{p^{a}}\right) \longrightarrow \pi_{*}^{\mathbf{S}}\left(S^{0}\right)\right] \supset\left(\operatorname{Im} J_{\mathbf{C}}\right)_{(p)} .
$$


Proof. Since $Z_{p^{a}} \subset S^{1}$, one can apply Proposition 2.5 for $K=\mathrm{C}$. Then one see that

$$
\operatorname{Im}\left[\phi: \pi_{*}^{\mathrm{s}}\left(B Z_{p^{a+}}\right) \longrightarrow \pi_{*}^{\mathrm{s}}\left(S^{0}\right)\right] \supset \operatorname{Im} J_{\mathrm{c}} .
$$

By Lemma $2.4,\left.\phi\right|_{\pi_{*}^{\mathbf{s}}\left(S^{0}\right)}(x)=p^{a}(x)$. Then clearly

$$
\left.\operatorname{Im} \phi\right|_{\pi_{*}} \mathbf{s}_{\left(B Z_{p^{a}}\right)} \supset\left(\operatorname{Im} J_{\mathbf{C}}\right)_{(p)} .
$$

q.e.d.

Let $Q\left(2^{a}\right)$ denote the generalized quoternionic group of order $2^{a+2}$. Then $Q\left(2^{a}\right) \subset S^{3}$ and we have similarly

Proposition 3.2. $\operatorname{Im}\left[\phi: \pi_{*}^{\mathrm{S}}\left(B Q\left(2^{a}\right)\right) \rightarrow \pi_{*}^{\mathrm{S}}\left(S^{0}\right)\right] \supset\left(\operatorname{Im} J_{\mathbf{H}}\right)_{(2)}$.

Now we prove Theorem 1.1, 1.2 and Proposition 1.3.

Proof of Theorem 1.1. Let $G$ be of $p$-rank 1. Let $i: G_{(p)} \rightarrow G$ be the inclusion of $p$-Sylow subgroup. Then the composition homomorphism

$$
\pi_{*}^{\mathbf{S}}\left(B G_{(p)+}\right) \stackrel{B i_{*}}{\longrightarrow} \pi_{*}^{\mathbf{S}}\left(B G_{+}\right) \stackrel{\phi G}{\longrightarrow} \pi_{*}^{\mathbf{S}}\left(S^{0}\right)
$$

is induced from adjoint map of the composite

$$
B G_{(p)+} \stackrel{B i}{\longrightarrow} B G_{+} \stackrel{B \rho}{\longrightarrow} B \Sigma_{|G|+} \stackrel{\omega}{\longrightarrow} Q\left(S^{0}\right) .
$$

Note that the restriction of the regular permutation of $G$ on $H$ is a direct sum of that of $H$. Thus we have a commutative diagram

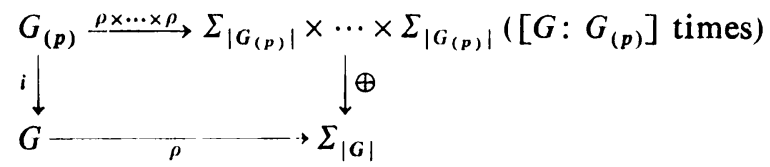

where $\oplus$ is the homomorphism defined by the juxtaposition. Thus we have

$$
\phi_{G}(B i)_{*}=\left[G: G_{(p)}\right] \phi_{G_{(p)}}
$$

Since $G$ is of $p$-rank $1, G_{(p)}$ is a cyclic group if $p$ is odd, and a cyclic group or a generalized quoternionic group if $p=2$. Then since $\left[G: G_{(p)}\right]$ is prime to $p$, the theorem follows from Proposition 3.1 and 3.2.

Proof of Theorem 1.2. Note that $\operatorname{Im} J=\operatorname{Im} J_{\mathbf{C}}=\operatorname{Im} J_{\mathbf{H}}$ in $\pi_{3}^{\mathbf{S}}\left(S^{0}\right)$, for canonical homomorphisms $\pi_{3}(S p) \rightarrow \pi_{3}(U) \rightarrow \pi_{3}(O)$ are isomorphisms. Therefore the only if part of the theorem follows from Theorem 1.1.

We now prove the if part. Suppose that the p-rank of $G$ is greater than 1 . Then $G$ contains a subgroup $H=Z_{p} \times Z_{p}$. Applying Lemma 2.3 for $G \supset H \supset e$, we obtain a commutative diagram 


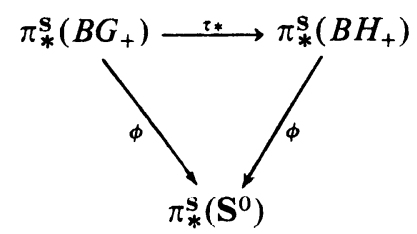

where $\tau_{*}$ is the transfer homomorphism induced from $\tau: Q\left(B G_{+}\right) \rightarrow Q\left(B H_{+}\right)$.

Suppose first that $p$ is odd. To prove the theorem, we have to show that $\phi$ : $\pi_{2 p-3}^{\mathbf{S}}(B G) \rightarrow \pi_{2 p-3}^{\mathbf{S}}\left(S^{0}\right)_{(p)}$ is not epimorphic. Assume contrary that $\phi$ is an epimorphism. Then by the above diagram,

$$
\phi: \pi_{2 p-3}^{\mathrm{S}}\left(B\left(Z_{p} \times Z_{p}\right)\right) \longrightarrow \pi_{2 p-3}^{\mathrm{S}}\left(S^{0}\right)_{(p)}
$$

is an epimorphism. Note that the regular permutation representation of $Z_{p} \otimes Z_{p}$ can be given by the composite

$$
Z_{p} \times Z_{p} \stackrel{\rho \times \rho}{\longrightarrow} \Sigma_{p} \times \Sigma_{p} \stackrel{\otimes}{\longrightarrow} \Sigma_{p^{2}}
$$

where $\rho$ is the regular permutation representation of $Z_{p}$ and $\otimes$ is defined by the standard action of $\Sigma_{p} \times \Sigma_{p}$ on $\{1, \ldots, p\} \times\{1, \ldots, p\} \cong\left\{1, \ldots, p^{2}\right\}$. Let $\gamma: \mathbf{S}\left(B\left(\Sigma_{p} \otimes\right.\right.$ $\left.\left.\Sigma_{p}\right)_{+}\right) \rightarrow \mathbf{S}$ be the stable map adjoint to

$$
B\left(\Sigma_{p} \times \Sigma_{p}\right)_{+} \stackrel{B \otimes}{\longrightarrow} B \Sigma_{p^{2}+} \stackrel{\omega}{\longrightarrow} Q\left(S^{0}\right)
$$

Then one has easily the following commutative diagram

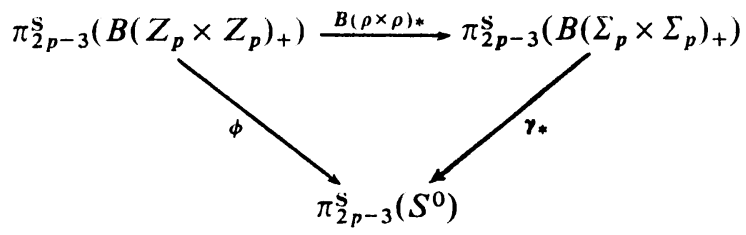

Note that $\left.\gamma_{*}\right|_{\pi_{2 p-3}^{\mathrm{s}}\left(S^{0}\right)}(x)=p x^{2}$ for $x \in \pi_{2 p-3}^{\mathrm{s}}\left(S^{0}\right) \subset \pi_{2 p-3}^{\mathrm{s}}\left(B\left(\Sigma_{p} \times \Sigma_{p}\right)_{+}\right)$. It is known [10] that $H_{*}\left(B \Sigma_{p}: Z_{p}\right)=0$ for $*<2 p-3$, and $H_{2 p-3}\left(B \Sigma_{p}\right)_{(p)}=Z_{p}$. Hence one see easily that $\pi_{2 p-3}^{\mathrm{s}}\left(B \Sigma_{p} \times B \Sigma_{p}\right)_{(p)} \cong Z_{p} \oplus Z_{p}$ generated by $j_{1 *}(u)$ and $j_{2 *}(u)$, where $j_{i}$ : $B \Sigma_{p} \rightarrow B \Sigma_{p} \times B \Sigma_{p}$ is the canonical inclusion, $i=1,2$, and $u \in \pi_{2 p-3}^{\mathbf{S}}\left(B \Sigma_{p}\right)$ is a generator. Let $d: \Sigma_{p} \rightarrow \prod^{p} \Sigma_{p}$ be the diagonal map. Then for $i=1,2$, the following diagram is commutative up to some inner automorphism of $\Sigma_{p^{2}}$.

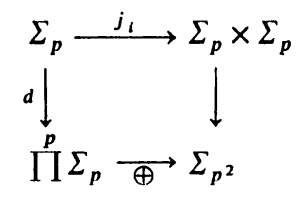

Remark that an inner automorphism induces the identity on stable homotopy groups. Then we see that

$$
\gamma_{*} j_{i *}=\mu_{*} d_{*}: \pi_{2 p-3}^{\mathrm{s}}\left(B \Sigma_{p+}\right) \longrightarrow \pi_{2 p-3}^{\mathrm{s}}\left(S^{0}\right)
$$


where $\mu: \mathbf{S}\left(B\left(\prod^{p} \Sigma_{p}\right)_{+}\right) \rightarrow \mathbf{S}$ is the adjoint of $\left.B\left(\prod^{p} \Sigma_{p}\right)_{+}\right) \stackrel{B \oplus}{\longrightarrow} B \Sigma_{p^{2}} \stackrel{\omega}{\longrightarrow} Q\left(S^{0}\right)$. Now it is easy to see that $\mu_{*} d_{*}(x)=p \phi(x)$ for $x \in \pi_{*}^{\mathbf{S}}\left(B \Sigma_{p+}\right)$. Hence $\gamma_{*} j_{i *}=0$ in $\pi_{2 p-3}^{\mathbf{S}}$ $\left(B \Sigma_{p+}\right)$ and this contradicts to the assumption. Hence $\phi: \pi_{2 p-3}^{\mathbf{S}}(B G) \rightarrow \pi_{2 p-3}^{\mathbf{S}}\left(S^{0}\right)$ is not epimorphism.

Next suppose that $p=2$. We have an isomorphism

$$
\pi_{3}^{\mathrm{s}}\left(B Z_{2} \times B Z_{2}\right) \cong \pi_{3}^{\mathrm{s}}\left(B Z_{2}\right) \oplus \pi_{3}^{\mathrm{s}}\left(B Z_{2}\right) \oplus \pi_{3}^{\mathrm{s}}\left(B Z_{2} \wedge B Z_{2}\right)
$$

By the homomorphism $\phi: \pi_{3}^{\mathbf{S}}\left(B Z_{2} \times B Z_{2}\right) \rightarrow \pi_{3}^{\mathbf{S}}\left(S^{0}\right)$, the first and the second summands are mapped onto $2 \pi_{3}^{\mathbf{S}}\left(S^{0}\right)_{(2)}$ by the same reason as for $p$ odd. Since $\pi_{3}^{\mathbf{S}}\left(S^{0}\right)_{(2)}$ $\cong Z_{8}$, it suffices to show that $\pi_{\mathbf{S}}^{3}\left(B Z_{2} \wedge B Z_{2}\right)$ contains no element of order 8 .

Let $M=S^{1} \cup_{2} e^{2}$ be the Moore space $\bmod 2$. Then $\pi_{3}^{\mathrm{S}}\left(B Z_{2} \wedge B Z_{2}\right) \rightarrow \pi_{3}^{\mathrm{S}}(M \wedge$ $M)$, for the 3-skeleton of $\mathbf{S} B Z_{2}$ is $\mathbf{S} M \vee \mathbf{S} S^{3}$. But it is easy to see that $\pi_{3}^{\mathbf{S}}(M \wedge$ $M) \cong Z_{4}$. This completes the proof.

q.e.d.

Proof of Proposition 1.3. Let $\varepsilon: \Sigma_{n} \rightarrow Z_{2}$ be the sign homomorphism. We easily see that $H_{1}\left(B \Sigma_{n}: \mathbf{Z}_{2}\right) \cong Z_{2}$, and $\varepsilon^{*}: H_{1}\left(B \Sigma_{n}: \mathbf{Z}_{2}\right) \rightarrow H_{1}\left(B Z_{2}: \mathbf{Z}_{2}\right)$ is an isomorphism for $n \geq 1$. Let $G$ be a finite 2-group. Then one can easily see that $\varepsilon \rho: G \rightarrow$ $Z_{2}$ is an epimorphism if and only if $G$ is a cyclic group, where $\rho: G \rightarrow \Sigma_{|G|}$ is the regular permutation representation. For if $G$ is not cyclic, for any $g \in G$, the restriction $\left.\varepsilon \rho\right|_{<g>}$ on the subgroup generated by $g$ is trivial. Therefore we see that

$$
\rho^{*}: H^{1}\left(B \Sigma_{2^{a}}: \mathbf{Z}_{2}\right) \longrightarrow H^{1}\left(B Z_{2^{a}}: \mathbf{Z}_{2}\right)
$$

is an isomorphism. Hence

$$
\rho_{*}: H_{1}\left(B Z_{2^{a}}\right) \longrightarrow H_{1}\left(B \Sigma_{2^{a}}\right) \cong H_{1}\left(Q S^{0}\right)
$$

is an epimorphism. This implies

$$
\phi: \pi_{1}^{\mathrm{s}}\left(B Z_{2^{a}}\right) \longrightarrow \pi_{1}^{\mathrm{s}}\left(S^{0}\right) \cong Z_{2}
$$

is an epimorphism. Then for a finite group $G$ with $G_{(2)} \cong Z_{2^{a}}(a>0)$, we see that

$$
\phi: \pi_{1}^{\mathrm{s}}(B G) \longrightarrow \pi_{1}^{\mathrm{S}}\left(S^{0}\right)
$$

is an epimorphism as in the proof of Theorem 1.1.

Next let $G$ be a finite group such that $G_{(2)}$ is not cyclic. Then

$$
\rho_{*}: H_{1}\left(B G_{(2)}\right) \longrightarrow H_{1}\left(B \Sigma_{2^{a}}\right)
$$

is trivial, and hence

$$
\phi: \pi_{1}^{\mathrm{S}}\left(B G_{(2)}\right) \longrightarrow \pi_{1}^{\mathrm{S}}\left(S^{0}\right)
$$

is trivial. Then so is $\phi: \pi_{1}^{\mathbf{S}}(B G) \rightarrow \pi_{1}^{\mathbf{S}}\left(S^{0}\right)$.

q.e.d.

\section{§4. Proof of Theorem 1.5}

Let $f: \mathbf{S} B Z_{2^{a}} \rightarrow \mathbf{S}, a \geq 2$ be a stable map. Let $\sigma \in \pi_{7}^{\mathrm{S}}\left(S^{0}\right)$ be the element of the 
Hopf invariant one. Suppose that there is an element $u \in \pi_{7}^{\mathbf{S}}\left(B Z_{2^{a}}\right)$ such that $f_{*}(u)$ $=\sigma$. Then since $\sigma$ is Hopf invariant one, we see easily that

$$
u_{*}: H_{7}\left(S^{7}: \mathbf{Z}_{2}\right) \longrightarrow H_{7}\left(B Z_{2^{a}}: \mathbf{Z}_{2}\right)
$$

is essential. Let $L^{n}\left(2^{a}\right)=S^{2 n+1} / Z_{2^{a}}$ be the standard lens space $\bmod 2^{a}$. Then $L^{n}\left(2^{a}\right)$ is the $2 n+1$ skeleton of $B Z_{2^{a}}$. The stable map $u: \mathbf{S}\left(S^{7}\right) \rightarrow \mathbf{S} B Z_{2^{a}}$ is then factored through a stable map

$$
u^{\prime}: \mathbf{S}\left(S^{7}\right) \longrightarrow \mathbf{S} L^{3}\left(2^{a}\right)
$$

such that

$$
u_{*}^{\prime}: H_{7}\left(S^{7}: \mathbf{Z}_{2}\right) \longrightarrow H_{7}\left(L^{3}\left(2^{a}\right): \mathbf{Z}_{2}\right)
$$

is an isomorphism. Let $\tau$ be the stable tangent bundle of $L^{3}\left(2^{a}\right)$. Then by the results of Atiyah [2] and by the mod $k$ Dold theorem [1], we see that $\tilde{J}(\tau) \in \tilde{J}\left(L^{3}\left(2^{a}\right)\right)$ is of odd order (may be zero). Thus in order to prove the theorem, it suffices to show the following

Lemma 4.1. Let $a \geq 2$ be an integer. Then $\tilde{J}(\tau) \in \tilde{J}\left(L^{3}\left(2^{a}\right)\right)$ is a non zero element of even order.

Proof. First we determine the tangent bundle of $L^{n}\left(2^{a}\right)$. Applying Theorem 1.1 of [14] to the principal bundle $Z_{2^{a}} \rightarrow S^{2 n+1} \rightarrow L^{n}\left(2^{a}\right)$, we see that

$$
\tau\left(L^{n}\left(2^{a}\right)\right) \oplus \varepsilon \cong(n+1) \eta
$$

where $\varepsilon$ is the trivial line bundle and $\eta=S^{2 n+1} \times{ }_{Z_{2^{a}}} \mathbf{C}^{1}$ is the canonical complex line bundle $\left(Z_{2^{a}}\right.$ acts on $\mathbf{C}^{1}$ via the canonical inclusion $\left.Z_{2^{a}} \subset S^{1}\right)$. Let

$$
i: L^{n}\left(2^{a}\right) \longrightarrow L^{n}\left(2^{a+1}\right)
$$

be the canonical map. Then it is obvious that $i^{*}\left(\tau\left(L^{n}\left(2^{a+1}\right)\right) \oplus \varepsilon\right) \cong \tau\left(L^{n}\left(2^{a}\right)\right) \oplus \varepsilon$. Hence we are enough to prove the lemma for $a=2$. Now it is known (Corollary 4.6, [8]) that the order of $\tilde{J}(r(\eta-1)) \in \tilde{J}\left(L^{3}(4)\right)$ is 8 . Thus

$$
\tilde{J}(\tau)=J(4 r(\eta-1))
$$

is an element of order 2. This completes the proof.

\section{Appendix}

The theory of infinite loop spaces says that a small category $\mathscr{C}$ with a coherent associative and commutative bifunctor $\bigotimes: \mathscr{C} \times \mathscr{C} \rightarrow \mathscr{C}$ (a symmetric monoidal category) defines a generalized cohomology theory ([9] and [13]). More precisely, if $\mathscr{C}$ is a symmetric monoidal category, one can associate a spectrum $\mathbf{B} \mathscr{C}=$ $\left\{B^{n} B \mathscr{C}\right\}_{n=0,1,2, \ldots}$ such that

i) $B^{0} B \mathscr{C}=B \mathscr{C}$ is the classifying space of $\mathscr{C}$

ii) $\left\{B^{n} B \mathscr{C}\right\}_{n \geq 1}$ is an $\Omega$-spectrum 
iii) if $B \mathscr{C}$ is of the homotopy type of countable $C W$ complex, then the structure map $g_{0}: B \mathscr{C} \rightarrow B^{1} B \mathscr{C}$ is the "group completion", i.e.,

$$
g_{0 *}: H_{*}(B \mathscr{C} ; k)\left[\pi_{0}(B \mathscr{C})^{-1}\right] \longrightarrow H_{*}\left(\Omega B^{1} B \mathscr{C} ; k\right)
$$

is an isomorphism for any field $k$. (see [9]).

The Barratt-Priddy-Quillen theorem asserts that the cohomology theory defined by the category of finite sets is equivalent to the stable cohomotopy theory (see [13]). Here we consider an equivariant version of the Barratt-Priddy-Quillen theorem, essentially due to Segal [12].

Let $\mathscr{C}$ be a symmetric monoidal category such that any morphism is invertible. Then $B \mathscr{C}$ is a homotopy commutative $H$-space, and the abelian (additive) monoid $\pi_{0}(B \mathscr{C})$ is identified with the set of isomorphism classes of $\mathrm{Ob} \mathscr{C}$. Given an object $X$, one has a functor

$$
L_{X}: \mathscr{C} \longrightarrow \mathscr{C}
$$

by $L_{X}(Y)=X \times Y$. This induces a continuous map

$$
l_{X}: B \mathscr{C} \longrightarrow B \mathscr{C}
$$

If $X$ and $Y$ are objects in the same component of $B \mathscr{C}$, then clearly $l_{X} \sim l_{Y}$ (homotopic). Let $\alpha \in \pi_{0}(B \mathscr{C})$. Choose a representative $X$ of $\alpha$, and put $\phi_{\alpha}=l_{X}$. Then clearly $\phi_{\alpha+\beta} \sim \phi_{\alpha} \phi_{\beta}$.

Now regard $\pi_{0}(B \mathscr{C})$ as a directed set by setting $\alpha<\beta$ if $\beta=\gamma+\alpha$ for some $\gamma$. Suppose that $\pi_{0}(B \mathscr{C})$ is countable. Then one can choose $d_{1}, d_{2}, \ldots, \in \pi_{0}(B \mathscr{C})$ such that the sequence $\left\{\alpha_{i}=d_{1}+\cdots+d_{i}\right\}_{i=1,2, \ldots}$ is cofinal in $\pi_{0}(B \mathscr{C})$. Consider the direct system

$$
B \mathscr{C} \underset{\phi_{\alpha_{1}}}{\longrightarrow} B \mathscr{C} \underset{\phi_{\alpha_{2}}}{\longrightarrow} B \mathscr{C} \longrightarrow \cdots
$$

it is clear that connected components of the direct $\operatorname{limit} \lim \left\{B \mathscr{C}, \phi_{\alpha_{l}}\right\}$ are homotopy equivalent to each other. So put

$$
B \mathscr{C}_{\infty}=\text { a component of } \lim \left\{B \mathscr{C}, \phi_{\alpha_{i}}\right\} .
$$

Theorem A.1. There is a map

$$
\omega: B \mathscr{C}_{\infty} \longrightarrow\left(\Omega B^{1} B \mathscr{C}\right)_{0}
$$

such that $\left.\omega_{*}: H_{*}\left(B \mathscr{C}_{\infty}\right) \rightarrow H_{*}\left(\Omega B^{1} B \mathscr{C}\right)_{0}\right)$ is an isomorphism, where the subscript 0 means the 0 -component.

Proof. Letting $S$ be the multiplicative subset of $\pi_{0}(B \mathscr{C})$ generated by $d_{1}, d_{2}, \ldots$,

$$
\begin{aligned}
& H_{*}(B \mathscr{C} ; k)\left[\pi_{0}(B \mathscr{C})^{-1}\right]=H_{*}(B \mathscr{C} ; k)\left[S^{-1}\right] \\
& \quad=\lim \left\{\rightarrow H_{*}(B \mathscr{C} ; k) \stackrel{\left(\phi_{\alpha_{i}}\right) *}{\longrightarrow} H_{*}(B \mathscr{C} ; k) \rightarrow \cdots\right\} \\
& \quad=H_{*}\left(\lim \left\{B \mathscr{C}, \phi_{\alpha_{i}}\right\} ; k\right) .
\end{aligned}
$$


for any field $k$ : For $\beta \in \pi_{0}(B \mathscr{C})$, let

$$
\omega_{\beta}: B \mathscr{C}_{\beta} \underset{g_{0}}{\longrightarrow}\left(\Omega B^{1} B \mathscr{C}\right)_{\beta} \underset{\phi-\beta}{\longrightarrow}\left(\Omega B^{1} B \mathscr{C}\right)_{0} .
$$

Then clearly $\omega_{\alpha+\beta} \phi_{\alpha} \sim \omega_{\beta}$, and hence by the telescope argument, we obtain a map

$$
\omega=\lim \omega_{\beta}: B \mathscr{C}_{\infty} \longrightarrow\left(\Omega B^{1} B \mathscr{C}\right)_{0} .
$$

The fact that $\omega^{*}$ is an isomorphism follows from that

$$
g_{0 *}: H_{*}(B \mathscr{C}: k)\left[S^{-1}\right] \longrightarrow H_{*}\left(\Omega B^{1} B \mathscr{C}: k\right)
$$

for any field $k$.

q.e.d.

Now let $G$ be a finite group. By $\mathscr{S}_{G}$ we denote the category of finite $G$-sets and $G$-isomorphisms. The direct sum and the direct product of finite $G$-sets give rise to binary functors $\oplus$ and $\otimes$, respectively. It is easy to see that $\left(\mathscr{S}_{G}, \oplus\right)$ and $\left(\mathscr{S}_{G}\right.$, $\otimes)$ are both symmetric monoidal categories.

Let $\Omega_{G}=\left\{\left(H_{1}\right), \ldots,\left(H_{k}\right)\right\}$ be the set of conjugacy classes of subgroups of $G$. Let $\mathscr{S}_{i}$ be the full subcategory of $\mathscr{S}_{G}$ consisting of objects of the form $n G / H_{i}=G / H_{i}$ $\cdots G / H_{i}$.

Lemma A.2. The category $\mathscr{S}_{G}$ is equivalent to the product category $\mathscr{S}_{1} \times \cdots$ $\times \mathscr{S}_{k}$, and

$$
B \mathscr{S}_{i}=\coprod_{n} E \Sigma_{n} \times_{\Sigma n}\left(B W_{H_{i}}\right)^{n}
$$

where $E \Sigma_{n}$ is a universal $\Sigma_{n}$-space and $W_{H_{i}}=N\left(H_{i}\right) / H_{i}$.

Proof. The first statement is clear. By definition $B \mathscr{S}_{i}=\coprod_{n} B \operatorname{Aut}_{G}\left(n G / H_{i}\right)$. Clearly we see that $\operatorname{Aut}_{G}\left(G / H_{i}\right)=W_{H_{i}}$. Hence $B \operatorname{Aut}_{G}\left(n G / H_{i}\right)=B\left(\Sigma_{n} \int W_{H_{i}}\right)=E \Sigma_{n} \times$ ${ }_{\Sigma n}\left(B W_{H_{i}}\right)^{n}$.

q.e.d.

Note that $B^{1} B \mathscr{S}_{i}=Q\left(\left(B W_{H_{i}}\right)_{+}\right)$by the Barratt-Priddy-Quillen theorem and by Lemma A.2. Thus we have obtained

Theorem A.3. There is a map

$$
\omega:\left(B \mathscr{S}_{G}\right)_{\infty} \longrightarrow \prod_{i=1}^{k} Q\left(\left(B W_{H}\right)_{+}\right)_{0}
$$

which induces an isomorphism of homology.

Next we shall consider subcategories of $\mathscr{S}_{G}$ which represent "free part" of $\mathscr{S}_{G}$. Let $\mathscr{F}_{G}$ and $\mathscr{F} \otimes$ be the fall subcategories of $\mathscr{S}_{G}$ consisting of free $G$-sets, and consisting of $G$-sets of the form free $G$ set $\cup+*$, respectively. Clearly $\mathscr{F}_{G}$ is a symmetric monoidal subcategory of $\mathscr{S}_{G}$, whereas $\mathscr{F} \otimes$ is symmetric monoidal with respect to $\otimes$.

Theorem A.4. There is a map

$$
\lambda:\left(B \mathscr{F} \mathbb{G}_{\infty} \longrightarrow Q_{0}\left(B G_{+}\right)_{P(G)}\right.
$$


inducing an isomorphism on homology, where $X_{P(G)}$ denotes the localization of $X$ at the set of primes $P(G)=\{p ; p|| G \mid\}$.

Proof. Note that $\pi_{0}\left(B \mathscr{F} \mathscr{G}^{\otimes}\right)$ is the multiplicative submonoid of $\pi_{0}\left(B \mathscr{S}_{G}\right)$ consisting of $1+n G$, where $G$ is regarded as a free $G$ set, hence $\pi_{0}\left(B \mathscr{F} \mathbb{G}_{G}\right)$ is a free abelian monoid with countable generators.

For $S, T \in \mathrm{Ob} \mathscr{S}_{G}$, let

$$
F(S, T): \mathscr{S}_{G} \longrightarrow \mathscr{S}_{G}
$$

be the functor defined by $F(S, T)(X)=S \oplus(T \otimes X)$. Let $f(S, T): B \mathscr{S}_{G} \rightarrow B \mathscr{S}_{G}$ be the induced map. Put $\gamma_{m}=f(m G, 1+m|G|)$. Then restricting to $B \mathscr{F}_{G}$, we have a map

$$
\gamma_{m}: B \mathscr{F}_{G} \longrightarrow B \mathscr{F}_{G}
$$

Also put $\beta_{m}=f(\phi, 1+m G): B \mathscr{F}_{G}^{\otimes} \rightarrow B \mathscr{F} \underset{G}{\otimes}$. Note that we have

$$
\begin{aligned}
(1+m G)(1+n G) & =1+(n+m+n m|G|) G \\
& =1+m G+(1+m|G|) n G
\end{aligned}
$$

in $\pi_{0}(B \mathscr{F} \underset{G}{\otimes})$. Hence we have a homotopy commutative diagram

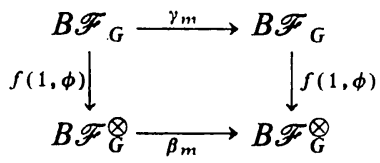

Similarly we have a homotopy commutative diagram

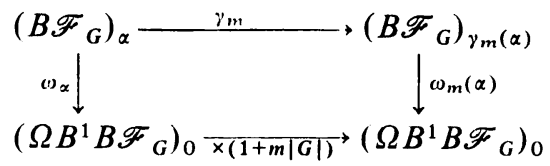

Note that if $m$ tends to infinity, then so does $\gamma_{m}(\alpha)$. Hence $\left(B \mathscr{F}_{G}\right)_{\gamma_{m}(\alpha)}$ approximates $\left(\Omega B^{1} B \mathscr{F}_{G}\right)_{0}$ homologically. Also note that the multiplicative set $M=\{1+$ $m|G| ; m=1,2, \ldots\}$ is cofinal in the multiplicative monoid of positive integers prime to $|G|$ because of the Dirichlet theorem on arithmetic progression.

Now since $\left(\Omega B^{1} B \mathscr{F}_{G}\right)_{0}$ is an $H$-space, the limit of

$$
\longrightarrow\left(\Omega B^{1} B \mathscr{F}_{G}\right)_{0} \overline{\times(1+m|G|)}\left(\Omega B^{1} B \mathscr{F}_{G}\right)_{0} \cdots
$$

is $\left(\left(\Omega B^{1} B \mathscr{F}_{G}\right)_{0}\right)_{P(G)}$ the localization at the set of primes $P(G)$. By Theorem A.3, $\left(\left(\Omega B^{1} B \mathscr{F}_{G}\right)_{0}\right)_{P(G)} \cong Q_{0}\left(B G_{+}\right)_{P(G)}$. Note that $\left(B \mathscr{F}_{G}^{\otimes}\right)_{\infty}$ is a component of $\lim _{m}\left\{B \mathscr{F} \mathbb{G}_{G}\right.$, $\left.\beta_{m}\right\}$, and

$$
f(1, \phi): B \mathscr{F}_{G} \longrightarrow B \mathscr{F}_{G}^{\otimes}
$$

is a homotopy equivalence. Hence taking the limit of the above diagrams, we have 
a map

$$
\lambda:(B \mathscr{F} \mathbb{G})_{\infty} \longrightarrow Q_{0}\left(B G_{+}\right)_{P(G)}
$$

which induces an isomorphism of homology.

q.e.d.

Finally we sketch briefly a reproof of Theorem 1.1 for odd primes using algebraic $K$-theory.

Let $\mathbf{F}_{q}$ be a finite field with $q=p^{r}$ elements. Let $\mathscr{V}$ be the category of (finite dimensional) vector spaces over $\mathbf{F}_{q}$ and linear isomorphisms. Let $\mathscr{V} \ll p>$ be the full subcategory of $\mathscr{V}$ consisting of vector spaces of a power of $p$ dimension. Let $\mathscr{S}_{\otimes}^{<p>}$ be the full subcategory of $\mathscr{S}$ (the category of finite sets) consisting of finite sets of cardinal $p^{n}$. Here the symmetric monoidal structures of $\mathscr{V}_{\otimes}^{<p>}$ and $\mathscr{S}_{\otimes}^{<p>}$ come from the tensor product and the direct product, respectively.

There are two functors

$$
\mathscr{V} \stackrel{U}{\longrightarrow} \mathscr{S}_{\otimes}^{<p>} \stackrel{L}{\longrightarrow} \mathscr{V} \stackrel{\otimes p>}{\longrightarrow}
$$

where $U$ forgets the vector space structure and $L$ sends a finite set to a vector space generated by the set.

Let $A$ be a subgroup of $\mathbf{F}_{q}^{*}$. By the scalar multiplication, $A$ acts on a $F_{q}$-vector space $V$ and the set $U(V)$. Since $\operatorname{Aut}_{F_{q}}(V)$ comutes with $A, U\left(\right.$ Aut $\left._{F_{q}}(V)\right) \subset$ Aut $_{A}$ $(U(V))$. . Note that $U(V) \in \mathrm{Ob} \mathscr{F} \mathbb{A}_{A}$. Thus we have a commutative diagram of functors

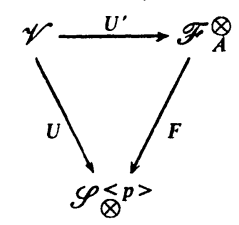

where $F$ is the functor forgetting the $A$-action.

Now we apply the functor $\Omega B^{1} B$ on the above categories. According to Quillen [11], $\left(\Omega B^{1} B \mathscr{V}\right)_{0} \cong B G L\left(\mathbf{F}_{q}\right)^{+}$and the algebraic $K$-group $\widetilde{K}_{\mathbf{F}_{q}}(X)$ is defined by $[X$, $\left.B G L\left(\mathbf{F}_{q}\right)^{+}\right]$. It is easy to see that $\left(\Omega B^{1} B \mathscr{V}_{\otimes}^{<p>}\right)_{0} \cong\left(B G L\left(\mathbf{F}_{q}\right)^{+}\right)_{<p>}$ and $\left(\Omega B^{1} B \mathscr{S}_{\otimes}^{<p>}\right)_{0}$ $\cong Q_{0}\left(S^{0}\right)_{<p>}$, where $X_{<p>}$ denotes the localization away from $p$. The functor $U$; $\mathscr{V} \rightarrow \mathscr{S}_{\circledast}^{<p>}$ induces a natural transformation

$$
j: \tilde{K}_{\mathbf{F}_{q}}(X) \longrightarrow\left[X, Q_{0}\left(S^{0}\right)_{<p>}\right] \cong \pi^{0}(X)\left[\frac{1}{p}\right]
$$

which refer to the algebraic $J$-homomorphism. Then by Theorem A.4, we have obtained

Proposition A.5. There is a commutative diagram

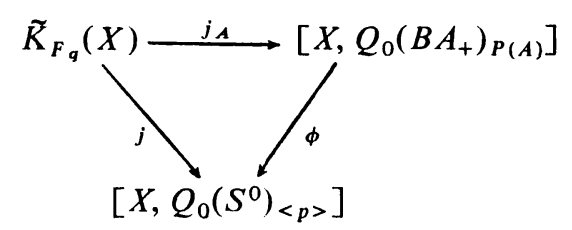


where $\phi$ is the forgetting homomorphism induced from $F$.

Now Theorem 1.1 for an odd prime follows from

Proposition A.6. Let $A=Z_{l^{a}}, l$ odd. Then there is an integer $q=p^{r}$ such that $\mathbf{F}_{q}^{*} \supset Z_{l^{a}}=A$ and

$$
j: \tilde{K}_{\mathbf{F}_{q}}\left(S^{2 n-1}\right)_{(l)} \longrightarrow \pi_{2 n-1}^{\mathbf{s}}\left(S^{0}\right)_{(l)}
$$

is an epimorphism onto $(\operatorname{Im} J)_{(l)}$.

Proof. The existence of such $q$ is well known. For $\operatorname{Im} j$, see Tornehave [15].

Department OF Mathematics KYOTO UNIVERSITY

\section{References}

[1] J. F. Adams, On the group $J(X)-I$. Topology 2 (1963), 181-195.

[2] M. F. Atiyah, Thom complexes. Proc. London Math. Soc., 11 (1961), 291-310.

[3] M. G. Barratt and S. Priddy, On the homology of non-connected monoids and their associated groups. Comment. Math. Helv. 47 (1972), 1-14.

[4] J. C. Becker and R. E. Schultz, Equivariant function spaces and stable homotopy theory. Comment. Math. Helv. 44 (1974), 1-34.

[5] H. Cartan and S. Eilenberg, Homological algebra. Princeton 1956.

[6] W. Feit and J. G. Thompson, Solvability of groups of odd order. Pac. J. Math. 13 (1963), $775-1029$.

[7] D. S. Kahn and S. Priddy, Applications of the transfer to stable homotopy theory. Bull. A.M.S. 78 (1972), 981-987.

[8] T. Kobayashi and M. Sugawara, On the stable homotopy types of stunted lens spaces. Hiroshima Math. J. 1 (1971), 287-304.

[9] J. P. May, $E^{\infty}$-spaces, group completions, and permutive categories. London Math. Soc. Lecture note 11 (1972).

[10] M. Nakaoka, Decomposition theorem for homology groups of symmetric groups. Ann. of Math. 71 (1960), 16-42.

[11] D. Quillen, On the cohomology and $K$-theory of general linear groups over a finite field. Ann. of Math. 96 (1972), 552-586.

[12] G. Segal, Equivariant stable homotopy theory. Actes. Congres Intern. Math. 2 (1970), 59-63.

[13] G. Segal, Categories and cohomology theories. Topology 13 (1974), 293-312.

[14] R. H. Szczarba, On tangent bundles of fibre spaces and quotient spaces. Amer. J. Math. 86 (1964), 685-697.

[15] J. Tornehave, The splitting of spherical fibration theory at odd primes, mineographed note, Aarhus.

[16] J. Wolf, Spaces of constant curveture. Publish or perish Inc., 1974.

[17] L. M. Woodward, The Kahn-Priddy theorem and equivariant vector fields on spheres. Comment. Math. Helv. 52 (1977), 1-10. 